\title{
LOS PROSPECTOS DE MEDICAMENTOS UNA HERRAMIENTA DIDÁCTICA PARA INCORPORAR ELEMENTOS DE TOXICOLOGÍA Y FARMACOLOGÍA EN EL PROFESORADO
}

\author{
Alicia E. Seferian $^{*}$ aliseferian@yahoo.com.ar
}

\section{Resumen}

La presente propuesta didáctica para los alumnos del profesorado, incorpora, a partir de la Química del Carbono, y Didáctica de la Química, elementos de toxicología y farmacología, mediante una visión sistémica interdisciplinar desde el enfoque Ciencia Tecnología y Sociedad (CTS), y ofrece una serie de posibles actividades que pueden desarrollarse en unidades especiales a partir del análisis de prospectos de medicamentos

\section{Palabras Clave}

Toxicología ,Farmacología, CTS, Tóxico, Prospecto

\footnotetext{
* Prof. Lic.

Didáctica Específica II y III de UNSAM

Química del carbono y Química y su Enseñanza del ISFD No 117 San Fernando

Enseñanza de las Ciencias Naturales I del IES N² Mariano Acosta Ciudad Autónoma Buenos Aires

Ex Capacitadora en los Equipos Técnicos Regionales (ETR) Región VI. Dir. ee Ed. Superior y Capacitación Educativa. Prov. Bs. As.
} 
El colectivo del profesorado del profesorado de Química -según se puede constatar en los cursos de capacitación docente-, así como en las evaluaciones de sus alumnos, mayoritariamente continúa enseñando con clases en su mayoría expositivas, escasamente dialogadas y contextualizadas, sin relaciones Ciencia-Tecnología-SociedadAmbiente; sin indagar los conocimientos reales que pudieran o no estar construyendo los estudiantes, y sin un replanteo de cómo revertir la escasa motivación y el generalizado rechazo a esta disciplina, como resultado de la enseñanza tradicional que recibieron en su formación. Seferian (2011)

Los futuros docentes del profesorado del siglo XXI, de la modalidad Química, requieren encarar la enseñanza de las ciencias con una mirada superadora de la tradicional que tenga a su vez una visión sistémica interdisciplinar desde la perspectiva Ciencia Tecnología y Sociedad (CTS).

La perspectivas CTS presenta consideraciones particulares entre las relaciones Ciencia, Tecnología y Sociedad muy apropiadas para la enseñanza que pueden resumirse en el llamado silogismo CTS, que según expresan Gordillo et al (2003) :

Premisas

* El desarrollo tecnocientífico es un proceso social como otros.

* El cambio tecnocientífico tiene importantes efectos en la vida social y en la naturaleza.

*Compartimos un compromiso democrático básico.
Las premisas remarcan los aspectos sociales implícitos en la ciencia y la tecnología y por otra parte focalizan en la cuestión de los movimientos sociales de los últimos años que fomentan la participación ciudadana en los temas tecnocientíficos.

Cabe destacar, que Gil Pérez (1993), señala al respecto: “... los diseñadores de programas reconocen que la ciencia sin sus implicaciones sociales, no significa mucho ni para los estudiantes ni para los ciudadanos".

La significatividad para su vida diaria de aquello que aprende, permite desarrollar en los estudiantes, una actitud positiva hacia las ciencias (Yager,1993; Penick,1992).

En este sentido, Acevedo (1996) resalta: "Como consecuencia del enorme trecho que hay entre los documentos de planificación de la enseñanza y la gestión de la misma en el aula, las metas que se formulan en los proyectos curriculares no predicen necesariamente posteriores actuaciones en clase. Muchos profesores, que son conscientes de los objetivos deseables, no saben luego cómo llevarlos a la práctica y continúan enseñando de la misma manera que siempre".

Es necesario que se incluyan los temas CTS en la formación inicial y permanente del profesorado, en diversas materias, para que sea posible contribuir más adecuadamente a mejorar e innovar en la enseñanza de las ciencias, con el fin de ayudar a todas las personas a conseguir una alfabetización científica y tecnológica más ajustada a sus necesidades. Acevedo (2002)

Resulta difícil, sin embargo, presentar nuevos enfoques desde una concepción de la disciplina rígida y pautada. 
Lograr superar esta visión, requiere un importante trabajo por parte del docente del profesorado para cambiar el encuadre sin perder la esencia misma de la materia y de este modo presentarles a los alumnos, situaciones innovadoras. No es posible esperar futuros docentes creativos con clases tradicionales y estereotipadas desde los centros de formación.

Podemos resumir en algunos puntos las ideas centrales, la presente propuesta que se comenzó a implementar en el ISFD No 117 en Química de Carbono y en Didáctica Específica II de la Licenciatura en Enseñanza de las Ciencias de la UNSAM:

$\checkmark$ Familiarizar a los docentes con los problemáticas aplicadas a nociones de toxicología y elementos de farmacología, desde una perspectiva CTS; $\checkmark$ Revalorizar la construcción escrita del conocimiento científico;

$\checkmark$ Adquisición de vocabulario específico de toxicología ambiental así como ciertos términos de farmacología que se encuentran en los prospectos de medicamentos;

- Ampliar la noción de interacciones hidrofílicas e hidrofóbicas desde elementos de toxicología.

$\checkmark$ Presentar un encuadre motivador desde la visión CTS, temas de química del carbono, relacionados con nociones de toxicología y elementos de farmacología que permitan a los futuros profesores adquirir una visión integrada de la Química.

- Diseñar unidades didácticas novedosas por parte de los alumnos del profesorado, con estas temáticas a fin de utilizarlas en futuras clases o en Práctica Docente.

\section{1.- El encuadre CTS en el profesorado}

Una de las líneas de investigación en Didáctica de las Ciencias estriba en el estudio de relaciones Ciencia, Tecnología y Sociedad (CTS), que- según se expresó anteriormente-,desde hace algunos años ha cobrado relevancia a nivel internacional. Al iniciarse el siglo XXI, nuestros alumnos, no pueden carecer de una formación que les posibilite la comprensión de cuestiones relacionadas con la ciencia y la tecnología ya que limitaría su capacidad de decisión frente a estas problemáticas contemporáneas y menos aun los futuros profesores de ciencias. Importantes proyectos de organismos internacionales como la UNES$\mathrm{CO}$ han presentado propuestas que contemplan las interacciones CTS (Gil-Pérez et al., 2005) a propósito de la Década de la Educación para el Desarrollo Sostenible.

\section{2.- Propuesta Didáctica}

La presente propuesta didáctica se llevó a cabo simultáneamente con 17 alumnos del $3^{\circ}$ año de Química del Carbono del profesorado $\mathrm{N}^{\circ} 117$ de Química de la Ciudad de San Fernando en la Provincia de Buenos Aires y 6 alumnos de Didáctica Específica II de la Licenciatura en Enseñanza de las Ciencias de la UNSAM.
A continuación se presenta la secuenciación de temas y de las actividades realizadas y por último, se realiza un análisis de los resultados obtenidos, así como una valoración personal de la propuesta. 
2.1.- Adquisición de terminología específica y relación de estos conceptos con información proveniente de los prospectos.

(2 horas, reloj)

Los prospectos presentan innumerable información que puede enriquecer el conocimiento de los alumnos del profesorado, ya que permite contextualizar diversos temas vistos en Química del Carbono y Química Biológica desde un encuadre CTS.

Resulta valioso que el futuro profesor pueda incorporar a su práctica, material relevante que tiene a su vez relación directa con la sociedad, ya que se trata de medicamentos prescriptos de manera frecuente por profesionales de la salud.

Los alumnos del nivel secundario se pueden interesar por la información proveniente de los prospectos, y los profesores pueden direccionar esta motivación con el fin de integrar y enriquecer aspectos propios de la disciplina con elementos de toxicología y farmacología.

En esta primera sección se trabajan, además de otros materiales, prospectos seleccionados que contengan términos que se desean explicitar como dosis, antagonismo, sinergismo, teratógeno entre otros.

Se realiza una actividad de exploración de preconcepciones a partir del siguiente enunciado:

"Discute con tus compañeros, ¿qué significación le damos en la vida diaria a términos como: tóxico, veneno, toxina, bioacumulación, dosis, antagonismo, sinergismo, teratógeno?".
Posteriormente, se realiza una puesta en común y se procede a la entrega de material específico de textos y prospectos seleccionados así como la red conceptual 1 para su análisis.

Se discuten, en pequeños grupos, las dificultades que presenta, por parte de alumnos de nivel secundario, la apropiación adecuada de dichos conceptos y algunas sugerencias para facilitar su aprendizaje.

2.2.- Discusión con respecto a la idea de toxicidad. (2 horas, reloj)

Se realiza una actividad de discusión a partir de los siguientes enunciados:

"En función de qué parámetros un medicamento o alguna sustancia e la vida diaria puede ser un toxico?; "El botox, utilizado para rejuvenecimiento facial, ¿Actúa como una toxina?. Investiga posibles riesgos"

2.3.- Biotransformación del tóxico en el organismo. (8 horas, reloj)

A partir de este momento se presentan los conocimientos disciplinares básicos referidos a la biotransformación del tóxico en el organismo que se encuentran resumidos en las redes conceptuales 1 y 2 . No se pretende profundizar al respecto, sino presentar esta temática de interés para el futuro profesor, a partir de las herramientas que le ofrece química del carbono y las nociones de química biológica. 
El tema se inicia, haciendo referencia a los dos procesos involucrados en la biotransformación: la Fase I, donde se generan moléculas más polares de xenobiótico, mediante reacciones biológicas, similares a las que ocurren en el laboratorio con la salvedad que se verifican en general en el RE (retículo endoplasmático) de las células y están mediadas por enzimas y transportadores de electrones
Las reacciones de Fase II, son en general reacciones de conjugación, denominadas así, ya que dichas moléculas se unen a compuestos endógenos (celulares) muy hidrosolubles catalizadas por enzimas específicas. Las reacciones de fase II sólo se verifican en los organismos vivos.

\section{Sustancia}

Quimica en

el ambiente

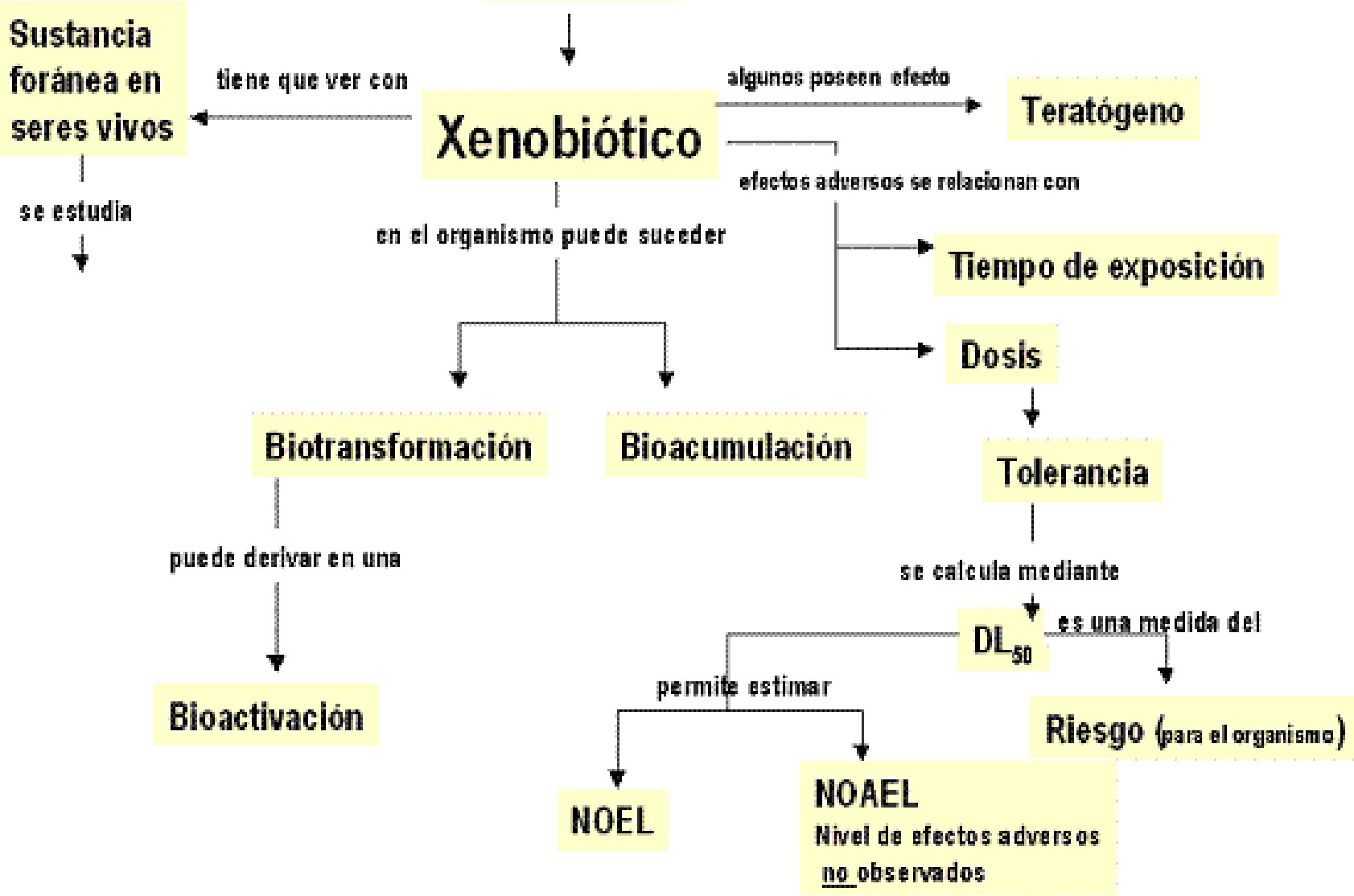

Ilustración 1 Xenobiótico: efectos 


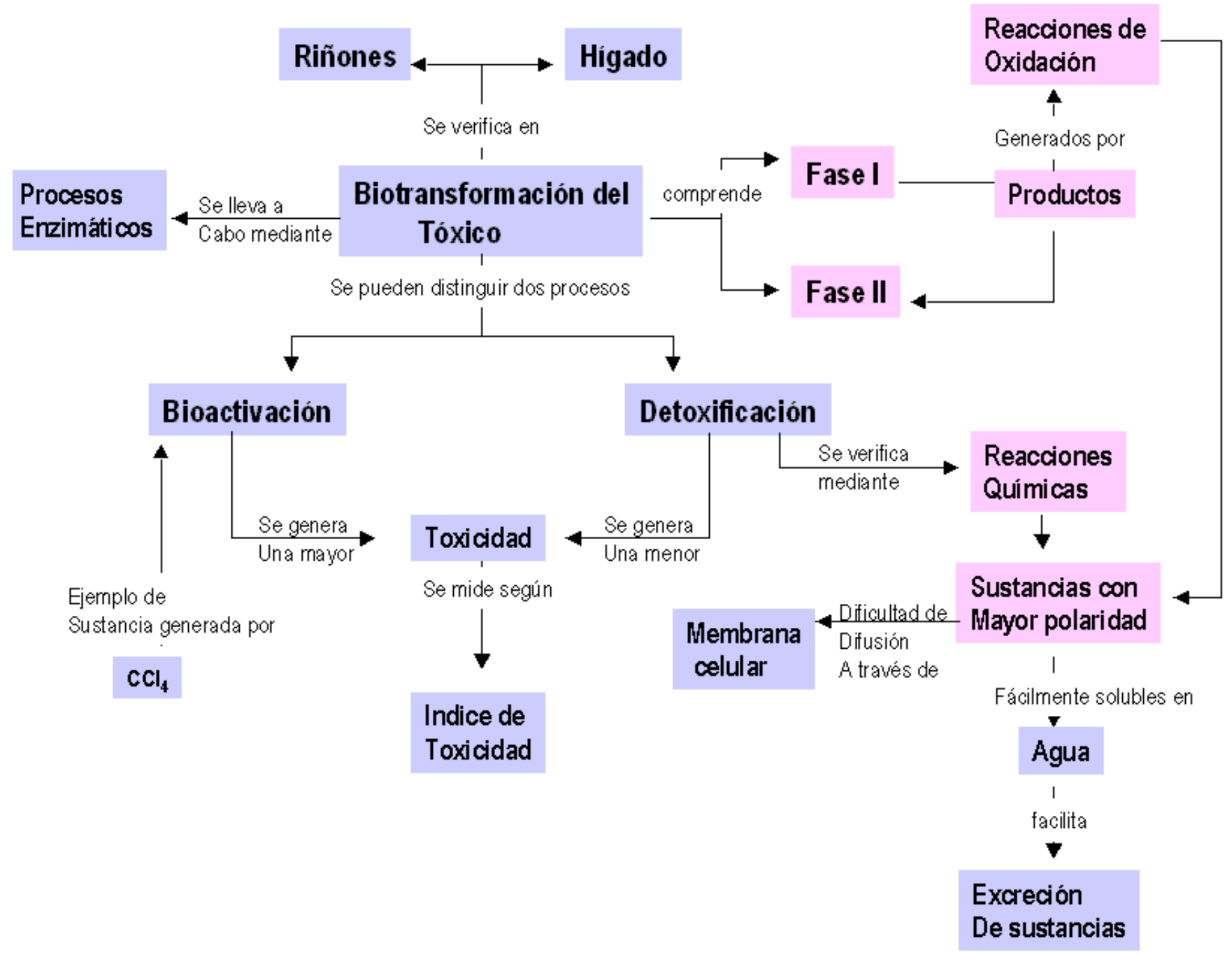

Ilustración 2 Biotransformación del tóxico en el organismo

En las reacciones de Fase II se agregan grupos polares a los productos de las reacciones de la Fase I en productos con menor actividad biológica y que pueden excretarse con mayor facilidad en la mayoría de los casos, según expresa Cockerman el al, (1994): "Phase II reactions involve the conjugation of the phase I products with an endogenous substance which usually renders the product less bioactive and more readly excreted".
La presencia de grupos polares en la molécula, por lo tanto, puede darle suficiente carácter hidrofílico para su rápida excreción. Para la mayor parte de las sustancias polares, sin embargo, este carácter no es suficiente y requieren una reacción subsiguiente a la de la Fase II para incrementar su solubilidad en agua, reacciones que suceden en la Fase II y permiten una rápida excreción a través de la orina. Las sustancias más comunes implicadas en la Fase II de conjugación son: ácido glucurónico, sulfato, glutatión, entre otros. 
Un ejemplo de estudio comparado de reacciones de biotransformación Fase I y reacción química similar, pero utilizando catalizadores en el laboratorio.

A continuación se presentan reacciones químicas muy similares, una de ellas analizada durante el año en el estudio de alcoholes, la segunda ecuación sin embargo presenta diferencias. Indica: dónde se producen (laboratorio, cualquier célula, de al- gún órgano determinado, sustancias que intervienen y función que cumplen y consecuencias que ocasiona (en el caso que se verificara en un organismo)"

Las ecuaciones químicas se representan en estos casos, mediante modelos moleculares con el fin de hacer hincapié en su configuración y no habituarnos a las fórmulas semi desarrolladas.
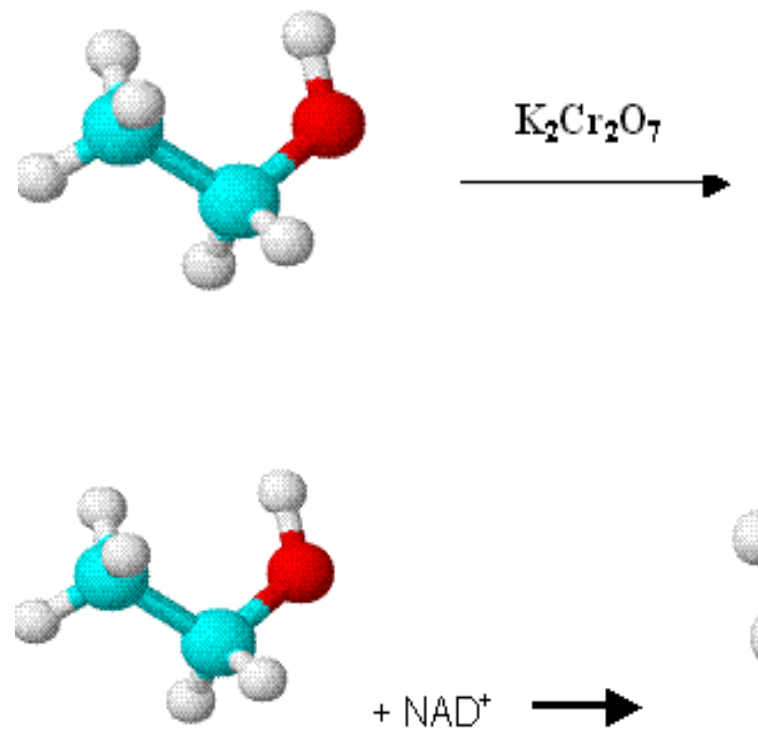

$$
+\mathrm{NAD}^{+}
$$

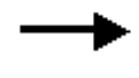

2.4- Integración de elementos de toxicología y farmacología en el análisis de un prospecto

Esta última parte de las actividades tiene que ver con integrar los conceptos de toxicología y reacciones de fase I y ll en el análisis de un prospecto y la adecuación al aula de dicha actividad para diferentes niveles de enseñanza, a partir de los 14 años en función del grado de complejidad.

Actividades sobre prospectos y su adecua-

$$
\text { ción al aula }
$$

A partir de diversos prospectos de medicamentos (antibióticos, mucolíticos, antitusivos, analgésicos entre otros), diseña una lámina informativa que indique la información relevante contenida en el mismo.

Discute con tus compañeros:

A-Qué podemos analizar de un prospecto con:

1- Alumnos de 14 años

2- Alumnos de 15 y 16 años

3- Alumnos de 17 años.

Justifica en cada caso, si es posible. 
B- Selecciona palabras o frases del prospecto y transcríbelas en la siguiente tabla, indicando nivel de complejidad y temas pa- ra relacionar con respecto a nociones de toxicología y hojas de seguridad, si es posible

\begin{tabular}{|r|l|l|l|}
\hline $\mathbf{N}$ & Palabras o frases & Nivel de complejidad apropiado para & Permite profundizar: \\
\hline 1 & & & \\
\hline 2 & & & \\
\hline
\end{tabular}

C- A continuación, se presentan posibles actividades diseñadas a partir de un prospecto, a modo de ejemplo.

Posteriormente a la lectura de las actividades, intenta diseñar guías de preguntas o problemas a partir de otro prospecto que hayas seleccionado y justifica su selección.

"Cada comprimido efervescente contiene 600 mg de N- acetilcisteína y diversos excipientes".

A partir de los datos que te presenta el prospecto, expresa la concentración del principio activo por cada $10 \mathrm{~g}$ de muestra y su composición centesimal. Busca información con respecto al instrumental actual requerido para medir la dosis correspondiente. ¿Qué importancia tiene ello?
¿Qué función cumplen los diversos excipientes? Recopila información y en función de sus propiedades trata de predecir el porqué de su variedad.

A continuación se transcribe un fragmento correspondiente a su Ac. Farmacológica: "La N-Acetilcisteína es un derivado de la cisteína, un aminoácido natural, que reduce la viscosidad del mucus bronquial, rompiendo por reducción los puentes bisulfuro de la fracción proteica de las glicoproteínas."

Escribe la fórmula semidesarrollada de la acetil cisteína y a partir de la siguiente representación de la estructura terciaria de una proteína, indica en ¿qué sitio se genera la reacción? y ¿qué producto esperarías obtener?
Segmento de polipéptido cuya estructura terciaria tiene origen en las interacciones moleculares

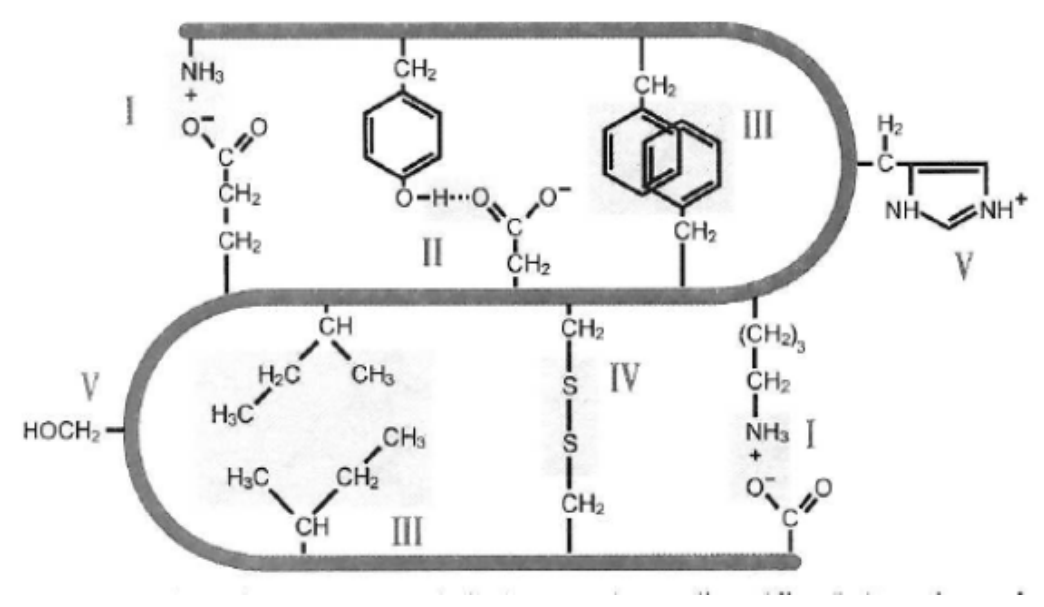

Tomado de: Blanco, A. Química biológica.

Bs. As. El Ateneo. $4^{\circ}$ edición 


\section{5.- Más información a partir de}

los prospectos.

En este caso se les solicita a los estudiantes que analicen los siguientes párrafos y los relacionen con los conceptos trabajados. Por otra parte, se indica que sugieran posibles actividades a partir del material presentado para cursos orientados en Ciencias Naturales en orden creciente de complejidad.

\section{AZITROMICINA}

"captada por fagocitos que migran a los sitios de infección..."

Vía de eliminación: hepatobiliar

\section{AMOXICILINA}

"se distribuye en líquidos biológicos"; "se une a proteínas plasmáticas"

Vías de eliminación: metabolitos glucurónidos conjugados hidrosolubles e inactivos" (es decir que se generaron reacciones de sustitución con formación de metabolitos más hidrosolubles)
SALBUTAMOL

$10 \%$ se une a proteínas del plasma. (agentes electrofílicos)

Vía de eliminación como sulfato fenólico biotransformación mediante unión a $\mathrm{SO}_{4}{ }^{-2}$ que incrementa su solubilidad.

\section{FLUTICASONA}

"La absorción por nariz es insignificante debido a la baja solubilidad en agua". "Se une en un 99\% a proteínas plasmáticas".

Vía de eliminación: "se metaboliza rápidamente en el hígado por medio del citocromo - 450 y se biotransforma en ácido carboxílico que se excreta a través de la bilis

\section{3.- Resultados}

Los resultados obtenidos, a partir de la presente propuesta de aula para el Nivel Superior que involucró a 17 alumnos del profesorado de la cátedra de Química del Carbono y por otra parte a 6 alumnos de la Licenciatura en Enseñanza de las Ciencias de la Universidad Nacional de Gral. San Martín de la cátedra de Didáctica Específica II, han sido un tanto dispares.

Se recopiló información acerca del conocimiento de terminología específica de elementos de farmacología y conjuntamente, se indagó, en el tipo de prospecto de medicamento seleccionado, para trabajar con los estudiantes del Nivel Medio, a partir de temas de química correspondientes a los diseños curriculares de $3^{\circ}, 4^{\circ}$ y $5^{\circ}$ año de la Escuela Secundaria (14, 15 y 16 años respectivamente) desde un encuadre CTS.

Por otra parte, se examinó la adecuación y pertinencia de las actividades de aula, a partir de los citados prospectos.

Finalmente, se solicitó una valoración personal con respecto al trabajo con la presente temática. 
3.1 Conocimiento de terminología específica.

\begin{tabular}{c|c} 
Estudiantes & $\begin{array}{c}\text { Conocimiento de la } \\
\text { terminología (\%) }\end{array}$ \\
\hline ISFD N ${ }^{\circ} 117$ & 10 \\
\hline UNSAM & 20 \\
\hline
\end{tabular}

La terminología empleada con respecto a elementos de farmacología y toxicología para trabajar esta propuesta didáctica se desconocía en la mayoría de los alumnos pese a que los conceptos presentados aparecen en prospectos de medicamentos así como también en revistas de divulgación científica y algunos programas radiales y televisivos de salud. Una de las posibles causas de ello tiene que ver con que estas temáticas no se vinculan de alguna manera con los contenidos del profesorado.

\section{2- Tipo de prospecto de medicamento seleccionado}

Los prospectos seleccionados fueron variados y algunos de uso veterinario ya que, según expresaron, las mascotas ocupan un lugar importante para los estudiantes de la escuela media.

\begin{tabular}{|c|c|c|}
\hline Medicamento & $\begin{array}{c}\text { ISDF N }{ }^{\circ} 117 \\
17 \text { estudiantes } \\
\%\end{array}$ & $\begin{array}{c}\text { UNSAM } \\
6 \text { estudiantes } \\
\%\end{array}$ \\
\hline Antitusivo & 10.0 & - \\
\hline $\begin{array}{l}\text { Complejo } \\
\text { Vitamínico }\end{array}$ & 17.6 & 33.3 \\
\hline Antinanauseoso & 5.0 & - \\
\hline Analgésico & 23.5 & 33.3 \\
\hline Antihistamínico & 17.6 & 16.6 \\
\hline Fitoterapéutico & 5.0 & 16.6 \\
\hline Antibiótico & - & 16.6 \\
\hline Antiviral & - & 16.6 \\
\hline Vasodilatador & 5.0 & - \\
\hline Antiácido & 10.0 & - \\
\hline $\begin{array}{l}\text { Sales de } \\
\text { Rehidratación oral }\end{array}$ & 5.0 & - \\
\hline
\end{tabular}

Los analgésicos, complejos vitamínicos y antialérgicos se seleccionaron en mayor proporción debido al mayor consumo de los mismos. Por otra parte los estudiantes justificaron dicha selección debido a los temas de Química que pueden relacionar. 


\section{3.- Palabras o frases relevantes, seleccionadas en prospectos.}

\section{A continuación se muestran las palabras o frases más relevantes seleccionadas por alumnos de la Licenciatura en Enseñanza de las Ciencias, para trabajar diferentes temas presentes en el DC}

\begin{tabular}{|c|c|c|c|}
\hline $\begin{array}{l}\text { Prospecto } \\
\text { de: }\end{array}$ & Palabras of frases & $\begin{array}{l}\text { Nivel de com- } \\
\text { plejidad apro- } \\
\text { piado para }\end{array}$ & Permite profundizar: \\
\hline antibiótico & $\begin{array}{l}\text { "la acción bactericida de las cefalosporinas } \\
\text { depende de la capacidad de alcanzar y unirse a } \\
\text { las proteínas(..)" }\end{array}$ & 15-16 años & $\begin{array}{l}\text { Interacciones moleculares hidrofílicas e } \\
\text { hidrofóbicas. }\end{array}$ \\
\hline anitusivo & suspensión & 14 años & soluciones \\
\hline antibiótico & $\begin{array}{l}\text { "(..) pueden producirse reacciones falso positi- } \\
\text { vas de glucosa en orina con soluciones de Feh- } \\
\text { ling y Benedict". }\end{array}$ & 17 años & $\begin{array}{l}\text { Hidratos de carbono. Oxidación de la gluco- } \\
\text { sa. }\end{array}$ \\
\hline antiviral & $\begin{array}{l}\text { "(el antiviral inhibidor de proteasas en el HIV) } \\
\text { ha demostrado inducir in vivo su propio meta- } \\
\text { bolismo y elevar la biotransformación de algu- } \\
\text { nas drogas metabolizadas por las enzimas del } \\
\text { citocromo P } 450 \text { y por glucuronización. }\end{array}$ & 17 años & $\begin{array}{l}\text { Biotransformación de tóxicos y reacciones } \\
\text { de conjugación. Interacciones moleculares } \\
\text { (aumento de la polaridad de la molécula } \\
\text { resultante) }\end{array}$ \\
\hline $\begin{array}{l}\text { complejo } \\
\text { vitamínico }\end{array}$ & Composición de una tableta efervescente & 14-15 años & composición centesimal. \\
\hline antibiótico & $\begin{array}{l}\text { Cada } 100 \mathrm{ml} \text { de gotas pediátricas, contiene } 5 \mathrm{~g} \\
\text { de cefalexina. }\end{array}$ & 14 años. & soluciones \\
\hline analgésico & $\begin{array}{l}\text { "(..)la mayoría de la dosis se recupera en la } \\
\text { orina bajo la forma de metabolitos, ibuprofeno } \\
\text { libre o conjugado". }\end{array}$ & 17 años & $\begin{array}{l}\text { Biotransformación de tóxicos y reacciones } \\
\text { de conjugación. Interacciones moleculares } \\
\text { (aumento de la polaridad de la molécula } \\
\text { resultante) }\end{array}$ \\
\hline \multirow[t]{2}{*}{$\begin{array}{l}\text { complejo } \\
\text { vitamínico }\end{array}$} & $\begin{array}{l}\text { "atención fenilcetonúricos: contiene fenilalani- } \\
\text { na" }\end{array}$ & 16 años & aminoácidos \\
\hline & $\begin{array}{l}\text { Dosis. Posología. Acción terapéutica. Excipien- } \\
\text { te. Principio activo. }\end{array}$ & 16-17 años & $\begin{array}{l}\text { Terminología específica básica de los pros- } \\
\text { pectos de medicamentos }\end{array}$ \\
\hline
\end{tabular}

\section{A continuación se presentan las palabras o frases más relevantes seleccionadas por estudian- tes del profesorado, para trabajar diferentes temas presentes en el DC:}

\begin{tabular}{|c|c|c|c|}
\hline Prospecto de: & Palabras of frases & $\begin{array}{l}\text { Nivel de com- } \\
\text { plejidad apro- } \\
\text { piado para }\end{array}$ & Permite profundizar: \\
\hline $\begin{array}{l}\text { complejo vita- } \\
\text { mínico }\end{array}$ & $\begin{array}{l}\text { "las vitaminas hidrosolubles del complejo B } \\
\text { y C" }\end{array}$ & 16-17 años. & $\begin{array}{l}\text { Interacciones moleculares. } \\
\text { Estructura molecular. }\end{array}$ \\
\hline \multirow[t]{2}{*}{$\begin{array}{l}\text { complejo vita- } \\
\text { mínico }\end{array}$} & $\begin{array}{l}\text { "atención fenilcetonúricos: contiene fenila- } \\
\text { lanina" }\end{array}$ & 16 años & aminoácidos \\
\hline & $\begin{array}{l}\text { Dosis. Posología. Acción terapéutica. Exci- } \\
\text { piente. Principio activo. Sobredosis. }\end{array}$ & 16-17 años & $\begin{array}{l}\text { Terminología específica básica de los pros- } \\
\text { pectos de medicamentos }\end{array}$ \\
\hline $\begin{array}{l}\text { complejo vita- } \\
\text { mínico }\end{array}$ & Contiene Fosfolípidos (lecitina) & 16- 17 años. & Biomoléculas. \\
\hline analgésico & $\begin{array}{l}\text { “(.) la dosis para caninos es de } 1-3 \mathrm{mg} / \mathrm{Kg} \\
\text { totales en el día. }\end{array}$ & 14 años & soluciones \\
\hline Vasodilatador & $\begin{array}{l}\text { Excipientes: celulosa microcristalina, Lacto- } \\
\text { sa anhidra, ácido silícico, almidón. }\end{array}$ & 16,17 años & Compuestos químicos \\
\hline Antihistamínico & $\begin{array}{l}\text { Ambos enantiómeros de fexofenadina, } \\
\text { desarrollaron efecto antihistamínico(...). }\end{array}$ & 17 años & estereoisomería \\
\hline $\begin{array}{l}\text { Suplemento de } \\
\text { hierro }\end{array}$ & $\begin{array}{l}\text { El hierro elemental se transforma a la for- } \\
\text { ma ferrosa por solubilización en el medio } \\
\text { ácido del estómago, }(. .)^{\circ}\end{array}$ & 17 años & Reacciones redox \\
\hline
\end{tabular}


Como se puede observar en las tablas anteriores, las palabras o frases seleccionadas son diversas así como los prospectos seleccionados. Sin embargo, la una gran parte de las palabras o frases no se relaciona con las reacciones de las Fases I y II y ello tiene que ver con la selección del prospecto adecuado, pero las relaciones explicitadas con respecto a temas de química son pertinentes.

Por otra parte, aunque la cantidad de estudiantes del profesorado es mucho mayor, varios de los trabajos presentados, no focalizaron en frases o palabras sino que realizaron generalizaciones sobre los posibles temas a trabajar, no se adecuaron a lo establecido.

En general, se consideran diversas frases y palabras que abarcan temas incluidos en los DCs de diversos años. Cabe destacar que los estudiantes de la Licenciatura son profesores que se han recibido hace algunos años por cuanto poseen experiencia de aula

\section{4.- Valoración personal de la propuesta.}

La valoración personal de la propuesta ha sido muy positiva y la mayoría de los estudiantes, tanto de la Licenciatura como los del profesorado, la han considerado de utilidad ya que son temas que desconocían y se relacionan de un modo apropiado con los contenidos de Química y Biología, por cuanto pueden trabajarse de un modo transversal a partir de las interacciones CTS.

Estudiantes del profesorado, sin embargo, consideran difícil seleccionar los prospectos adecuados ya que carecen del conocimiento necesario.

Por otra parte, algunos pocos estudiantes del profesorado con una visión de la enseñanza tradicional, piensan sin embargo que son temas que no corresponde tratar ya que no se encuentran en el DC de la escuela secundaria.

\section{Consideraciones finales.}

La presente propuesta didáctica, encarada desde una visión sistémica interdisciplinar a partir de la perspectiva Ciencia Tecnología y Sociedad (CTS), se ha presentado en forma abreviada en función de elementos de toxicología y farmacología y ha tenido como finalidad, mostrar un sinnúmero de posibilidades que pueden desarrollarse en el profesorado, desde la Química del Carbono y su relación con Química Biológica, así como en Didáctica Específica II de la Licenciatura en Enseñanza de las Ciencias, para profesores.

La mayoría de los estudiantes intentan cambiar en sus prácticas el encuadre tradicional de las clases de química, pero comentan que les resulta sumamente difícil, por cuanto se hace necesario que ello se verifique a partir de las materias específicas que se cursan en la carrera, como lo han solicitado.

Por otra parte, resulta de interés para la comunidad informarse con respecto a medicamentos de venta libre así como de los entes nacionales reguladores de medicamentos, a fin de tener una idea de aquello que se consume.

Para concluir, las temáticas presentadas han sido recibidas con agrado por estudiantes que requerían información al respecto, dado que se trata de temas de actualidad, relacionados con la ciencia y la sociedad, que se presentan en los medios masivos de comunicación. 


\section{Bibliografía}

Acevedo, J., Vázquez Alonso, A., Manassero M., y Acevedo Romero, P. (2002) Persistencia de las actitudes y creencias CTS en la profesión docente. Revista Electrónica de Enseñanza de las Ciencias, Vol. 1, № 1, 1-27

Acevedo, J. Cambiando la práctica docente en la enseñanza de las ciencias a través de CTS. Borrador, 13, 26-30. 1996. consultada por última vez en noviembre, 2007 de la URL www.campusoei.org/salactsi/acevedo2.htm

Campanario, Juan, M. y Moya, A. ¿Cómo enseñar ciencias? Principales tendencias y propuestas. Enseñanza de las Ciencias 17 (2) pps. 179-192. 1999.

Cockermann, Lorris, G. y Shane, B. Basic Environmental toxicology. Florida. U.S.A. Ed. Florida. CRC Press.1994.

Chang, R. Química. Ciudad de México. México. Mc. Graw Hill. 1994.

Galagovsky, L. R. Redes conceptuales: aprendizaje, comunicación y memoria. Buenos Aires. Argentina. Lugar Editorial.1999.

Gómez Moliné, M. Reflexiones sobre el lenguaje de la ciencia y el aprendizaje. Educación Química 11(2) 266-273.1999.

Gilbert, J.,(2005) Constructing words through science education. The selected works of John Gilbert. WLE World Library of Educationalists.

Hodson, D. Hacia un enfoque más crítico del trabajo de laboratorio. Enseñanza de las ciencias, 12 (3) pp. 299-313. 1994.

Ibáñez, V. y Gómez Alemany, I La interacción y la regulación de los procesos de enseñanza aprendizaje en la clase de ciencias: análisis de una experiencia. Enseñanza de las Ciencias 23 (11), 97-110 Barcelona ICE. 2005.

Lemke, J. Aprender a hablar ciencias. Bs. As. Argentina. Ed. Paidós. 1997.

Loomis, T. Fundamentos de toxicología Barcelona. España. Ed. Acribia. 1982 


\section{Bibliografía}

López Cerezo, J. A. "Bibliografía Básica sobre CTS", consultada por última vez en diciembre 15, 2008 de la URL <http//www.campus-oei.org/cts/bibliografía.htm>

Monereo Font, C. Enseñar a conciencia. Hacia una didáctica metacognitiva. En: Galagovsky, L., Bonán, L. y Adúriz Bravo, A. Problemas con el lenguaje científico en la escuela. Un análisis desde la observación de clases de ciencias naturales. Enseñanza de las Ciencias, 16 (2), pp. 315-321. Barcelona: ICE.1998

Novak,J. D. y Gowin, B. Aprendiendo a aprender Barcelona. España. Ed. M. Roca.1998.

Seferian, A. (2011) La enseñanza de la química en el nivel de profesorados. Reflexiones para el siglo XXI. Cap. 38. Pp. 289-294. En: La Química en la Argentina. Texto editado por la Asociación Química Argentina y coordinado por la Dra. Lydia Galagovsky a propósito del centenario de la institución. (En prensa).

Vilches, A. Y Furió, C.: Ciencia Tecnología y Sociedad: Implicaciones en la educación científica del siglo XXI. Una versión electrónica de este artículo fue consultada por última vez en diciembre 15, 2008 de la URL http://www.campus-oei.org/cts/ ctseducacion.htm.-

Gil Pérez, D. y Guzmán Ozámiz, M. (1993) Enseñanza de las Ciencias y la Matemática: tendencias e innovaciones. Editorial Popular. Madrid

Yager, R. (1993) "The advantages of STS approaches in science instruction in grades four through nine". Bulletin of Science, Technology and Society, 13, pp. 74-82. en García Palacios, E. López Cerezo, J. et al (2001) Ciencia Tecnología y Sociedad: una aproximación conceptual. OEI Cuadernos de Iberoamérica. Madrid

Penick, J. (1992): "STS. Instruction enhances student creativity, en Yager (1992): The status of science-technology- society. Reforms around the world International Council of Associations for Science Education/Yearbook. 